\title{
Резонансные оптические покрытия, состоящие из массивов частиц SiGe и Ge на подложках $\mathrm{Si}$
}

\author{
А.А. Шкляев ${ }^{1,2}$ \\ ${ }^{1}$ Институт физики полупроводников им. А.В.Ржанова СО РАН, \\ Новосибирск, 630090, пр. Ак. Лаврентьева, 13 \\ ${ }^{2}$ Новосибирский государственный университет, Новосибирск, 630090, ул. Пирогова, 2 \\ тел: +7 (913)001-0085, эл. почта: shklyaev@isp.nsc.ru
}

DOI $10.34077 /$ RCSP2021-26

Эффективность оптоэлектронных элементов в значительной мере определяется свойствами их поверхности по отношению к отражению и пропусканию электромагнитного (ЭМ) излучения в используемом спектральном диапазоне. В последнее десятилетие на смену традиционным плоским антиотражающим покрытиям приходят покрытия, состоящие из массивов металлических и диэлектрических частиц субволнового размера. Их действие основано на возбуждении в них плазмонных или ЭМ резонансов соответственно. По сравнению с покрытиями, состоящими из плоских слоёв, их преимущества состоят в отражающей и пропускающей способности, практически не зависящей от угла падающего ЭМ излучения, которая реализуется в более широком спектральном диапазоне.

Сравнение между собой покрытий, состоящих из металлических и диэлектрических частиц, показывает, что плазмонные резонансы создают вокруг частиц локальные ЭМ поля более сильные, чем магнитные и электрические резонансы, возбуждаемые в диэлектрических частицах. В то же время возбуждение плазмонных колебаний сопряжено с сильной диссипацией падающего излучения, тогда как возбуждение магнитных и электрических резонансов определяется коэффициентами поглощения диэлектрических материалов и может быть незначительным в выбранном спектральном диапазоне. Сравнение свойств антиотражающих покрытий из металлических и диэлектрических частиц было проведено в работе [1]. Оно показало, что покрытие из наночастиц серебра может увеличить общее поглощение на $30 \%$ в полупроводниковой подложке, тогда как покрытие из частиц кремния позволяет увеличить поглощение на $64 \%$, но в более узкой спектральной полосе за счёт опосредованного подложкой эффекта Керкера. При этом спектральное положение полосы можно задавать выбором размеров наночастиц.

Важным аспектом покрытий из массива частиц, определяющим их свойства, является метод их получения. Один из наиболее простых методов основан на использовании эффекта несмачивания подложки осаждаемым на неё материалом. Он может быть применён для получения покрытий как из металлических, так и из диэлектрических частиц [2]. Этот метод позволяет получать покрытия на больших площадях, однако обычно приводит к получению неупорядоченных частиц с широким распределением их по размеру. Антиотражающие свойства таких покрытий характеризуются широким спектральным диапазоном [3].

Массивы упорядоченных частиц заданного размера и формы могут быть получены с использованием различных литографий, в частности, электронной [4]. Такая возможность позволяет экспериментально изучать влияние на оптические свойства таких параметров, как расстояние между частицами и их расположение относительно друг друга, то есть решёточные эффекты, а также влияние формы частиц.

Экспериментальные работы в данной области часто опережаются теоретическими исследованиями. Взаимодействие ЭМ волн с диэлектрическими частицами субволнового размера не имеет простой интерпретации для описания спектров отражения и пропускания. Единственным простым оценочным соотношением является формула $\lambda_{\mathrm{m}}=n d$, где $\lambda_{\mathrm{m}}$ - длина волны магнитного дипольного резонанса, $n-$ показатель преломления материала частицы и $d$ - диаметр частицы. Была показано, что эта формула с высокой точностью выполняется для сферических частиц в среде воздуха. Для расчётов более сложных случаев могут быть использованы коммерческие программные пакеты.

\section{Лumepamypa}

[1] K.V. Baryshnikova, et al.// Sci. Rep. 2016. No.6. Art no. 22136.

[2] A.A. Shklyaev and A.V. Latyshev// Sci. Rep. 2020, no.10. Art no.13759.

[3] A.A. Shklyaev and A.V. Tsarev// IEEE Photonics J. 2021. Vol. 13, no.3. Art no.2200212.

[4] D.E. Utkin, et al. // Opt. Mater. 2020. Vol.109. Art no. 110466. 